\title{
Theory of thermal nonequilibrium entropy in near-field thermal radiation
}

\author{
Arvind Narayanaswamy* and Yi Zheng \\ Department of Mechanical Engineering, Columbia University, New York, New York 10027, USA \\ (Received 6 November 2012; revised manuscript received 28 June 2013; published 12 August 2013)
}

\begin{abstract}
We propose a theoretical formalism to evaluate the entropy density and entropy flux that takes into account near-field effects, i.e., interference, diffraction, and tunneling of waves. Using the fluctuation-dissipation theorem, expressions for entropy density and entropy flux in a vacuum cavity between planar multilayered media are derived in terms of local density of photons, local density of accessible microscopic states, and velocity of energy transmission. The proposed method is used to determine the maximum work that can be extracted and a thermodynamic limit of the energy conversion efficiency that can be obtained in near-field thermal radiation.
\end{abstract}

DOI: 10.1103/PhysRevB.88.075412

PACS number(s): 44.40.+a, 05.70.Ln, 42.25.-p

\section{INTRODUCTION}

The inception of modern quantum physics can be traced back to Planck's pioneering work on blackbody radiation. ${ }^{1}$ Central to Planck's work is the thermodynamic analysis of thermal radiation in a cavity, which requires knowledge of energy, momentum, and entropy of photons. Planck's analysis is restricted to the case when near-field effects, i.e., the collective influence of diffraction, interference, and tunneling of waves, are absent. ${ }^{1}$ When all objects are at the same temperature, i.e., in thermal equilibrium, thermodynamic analysis can be completed by knowledge of the pressure or stress tensor alone because changes in Helmholtz free energy can be equated to the $p d V$ work done in compression or expansion. From Helmholtz free energy, other quantities of interest, like entropy, can be derived. In thermal nonequilibrium, Helmholtz free energy can no longer be used and we need to know the entropy due to near-field effects in addition to the stress tensor. Though enhancement of radiative transfer due to near-field effects has been proposed as a method of increasing the power density of thermophotovoltaic devices, ${ }^{2-4}$ thermodynamic analysis is absent from literature because of, primarily, the lack of knowledge of entropy transfer between two objects at different temperatures when near-field effects are important.

Though the near-field radiative energy and momentum transfer are well known, ${ }^{5,6}$ little is known about entropy associated with near-field radiative transfer. ${ }^{7}$ In the intervening six years, not much has changed with respect to our knowledge of entropy transfer in the presence of near-field effects. In this paper, we show how near-field entropy density and flux in a planar vacuum cavity between two half-spaces at different temperatures can be determined. Using the entropy flux, we predict the maximum work that can be extracted through near-field radiative transfer between two half-spaces at different temperatures. In developing the theory of entropy density and entropy flux, we have defined two new concepts that will, hopefully, prove useful elsewhere too. They are (1) the concept of local density of accessible microscopic states, and (2) generalized spectral specific intensity, which enables usage of the concept of specific radiative intensity ${ }^{1,8,9}$ for propagating as well as evanescent waves.

First, we review a subset of the works on entropy density and flux of thermal radiation relevant to this paper. For blackbody radiation at temperature $T$, the far-field spectral entropy intensity of radiation (per polarization) is given by (see Eq. 278 of Planck ${ }^{1}$ )

$$
\begin{aligned}
l_{\omega}(\hat{\Omega})= & \frac{k_{B} \omega^{2}}{8 \pi^{2} c^{2}}\left[\left(1+\frac{I(\omega, \hat{\Omega})}{\hbar \omega^{3} / 8 \pi^{2} c^{2}}\right) \ln \left(1+\frac{I(\omega, \hat{\Omega})}{\hbar \omega^{3} / 8 \pi^{2} c^{2}}\right)\right. \\
& \left.-\left(\frac{I(\omega, \hat{\Omega})}{\hbar \omega^{3} / 8 \pi^{2} c^{2}}\right) \ln \left(\frac{I(\omega, \hat{\Omega})}{\hbar \omega^{3} / 8 \pi^{2} c^{2}}\right)\right],
\end{aligned}
$$

where $k_{B}$ is Boltzmann constant, $c$ is the speed of light in free space, $\omega$ is the angular frequency, $2 \pi \hbar$ is the Planck constant, and $I(\omega, \hat{\Omega})$ is the spectral radiation intensity of a ray in the direction defined by unit vector $\hat{\Omega}$. The spectral entropy density is given by

$$
s_{\omega}=\frac{1}{c} \int l_{\omega}(\hat{\Omega}) d \Omega .
$$

The assumptions in Eqs. (1) and (2) are (1) the speed of light is given by its value in free space, and (2) the density of states per polarization is given by its value in free space $\left(\omega^{2} / 2 \pi^{2} c^{3}\right)$.

Petela $^{10}$ used the concept of exergy or availability of unpolarized uniform radiation to calculate the maximum work that can be extracted from two thermal reservoirs interacting through thermal radiation. His approach relied on defining exergy flux in terms of far-field entropy intensity as follows:

$$
b(T)=\frac{\sigma}{3} T^{4}+2 \pi \int I_{\omega}(T) d \omega-2 \pi T \int l_{\omega}(T) d \omega,
$$

where $b$ is the exergy of radiation, $\sigma$ is the Stefan-Boltzmann constant, and $I_{\omega}$ and $l_{\omega}$ are isotropic energy and entropy intensity, respectively. Landsberg and Tonge ${ }^{11}$ introduced the concepts of dilute blackbody radiation and effective temperature in order to determine the amount of work that can be extracted from solar radiation (see Sec. V for further details of Landsberg and Tonge ${ }^{11}$ ).

The first investigation of entropy of interfering electromagnetic beams can be attributed to von Laue. ${ }^{12}$ Since the original paper is in German, we rely on Nigam's work, ${ }^{13}$ which is based on von Laue's work but is in English. Relying on Planck's expression for entropy intensity, von Laue's surprising conclusion was that interference effects could lead to a decrease in the total entropy of two interfering beams. The entropy of $N(N \geqslant 2)$ partially coherent pencils of radiation was investigated by Barakat and Brosseau. ${ }^{14,15}$ The $N=2$ case was investigated earlier by von Laue. ${ }^{12}$ The key result of 
the works of von Laue and Barakat and Brosseau useful for this paper is that the total entropy of two incoherent pencils of radiation is the sum of the entropies of the two pencils. There have been other works also expounding on the entropy of far-field thermal radiation, ${ }^{7,16-19}$ all of which rely on Planck's theory of thermal radiation.

The position dependence of energy and entropy density due to near-field effects in thermal equilibrium was investigated for the first time by Dorofeyev. ${ }^{20}$ In his work, the $\omega^{2} / \pi^{2} c^{3}$ is replaced by the local density of states, which is position dependent. That Dorofeyev's method is valid only at equilibrium can be seen from the relation for spectral entropy density, ${ }^{20}$ which is

$$
s_{\omega}(\boldsymbol{r}, T)=\rho_{\omega}(\omega, \boldsymbol{r}) \frac{\partial}{\partial T}\left[k_{B} T \ln (Z(\omega, T))\right],
$$

where $\rho_{\omega}(\omega, \boldsymbol{r})$ is the position dependent local density of states and $Z(\omega, T)=\exp \left(-\hbar \omega / 2 k_{B} T\right) /\left[1-\exp \left(-\hbar \omega / k_{B} T\right)\right]$ is the equilibrium partition function. As we will show in Sec. V, the entropy flux in thermal equilibrium is identically equal to zero (as is energy flux). We should also mention that PerezMadrid et al. ${ }^{21-23}$ have treated energy transfer between two objects under the framework of mesoscopic nonequilibrium thermodynamics. Although the word "entropy"appears a few times in their works, it has little to do with the near-field entropy density or flux in this study. Their work deals mainly with heat exchange between two nanoparticles which can change configuration in the near field. ${ }^{21}$ The mesoscopic entropic formalism was introduced to take into account the changes in particle shape and structure and not the thermally generated electromagnetic waves themselves. With the exception of Dorofeyev's work, ${ }^{20}$ most works on entropy of thermal radiation make the assumption that the local density of states and velocity of radiation are independent of position and given by their values in free space. These assumptions break down when near-field effects are present and, to the best of our knowledge, have not been investigated under conditions of thermal nonequilibrium.

The structure of the paper is as follows. In Sec. II, elements of fluctuational electrodynamics necessary to determine entropy density and entropy flux are discussed. In Sec. III we derive an expression for entropy density of near-field thermal radiation in a vacuum cavity between two planar multilayer objects. The concept of accessible microscopic states is also discussed here. In Sec. IV, we define the concept of generalized radiative intensity, and use it to determine entropy flux. In Sec. V, we show that the entropy density and flux as formulated here agrees with Planck's blackbody radiation theory. We also use the entropy flux in order to determine the maximum efficiency of conversion of heat to work. Our work is summarized in Sec. VI.

\section{THEORETICAL FUNDAMENTALS}

Rytov's theory of fluctuational electrodynamics ${ }^{24}$ can be thought of as a combination of statistical physics, quantum physics, and macroscopic electrodynamics. The fluctuationdissipation theorem ${ }^{25,26}$ is used to relate the power spectral density of the fluctuating charge density to the local temperature, frequency dependent relative dielectric permittivity $[\varepsilon(\omega)]$, and relative magnetic permeability $[\mu(\omega)]$ of the object. For vacuum $\varepsilon(\omega)=\mu(\omega)=1$ for all $\omega$. The electromagnetic fields generated because of any charge distribution can be written as integrals of the electric and magnetic dyadic Green's functions, $\overline{\overline{\mathbf{G}}}_{e}\left(\omega ; \mathbf{r}, \mathbf{r}^{\prime}\right)$ and $\overline{\overline{\mathbf{G}}}_{m}\left(\omega ; \mathbf{r}, \mathbf{r}^{\prime}\right){ }^{6,27,28}$ The details of the dyadic Green's functions, such as the boundary conditions and reciprocity relations they satisfy, are given in the following references. ${ }^{6,28}$ Since we are concerned with fluctuational phenomena, the ensemble averages of products of field components $\mathbf{E}(\omega, \mathbf{r})$ and $\mathbf{H}(\omega, \mathbf{r})$ are of interest to us. For instance, spectral energy transfer between two objects can be obtained from the ensemble averaged spectral Poynting vector: ${ }^{29}$

$$
P_{i}(\omega, \mathbf{r})=\operatorname{Re}\left(\epsilon_{i j k}\left\langle E_{j}(\omega, \mathbf{r}) H_{k}^{*}(\omega, \mathbf{r})\right\rangle\right),
$$

where $P_{i}$ is the $i$ th component of the Poynting vector $\mathbf{P}$ ( $i=1,2,3$ or $x, y, z)$, and $\epsilon_{i j k}$ is the Levi-Cevita symbol. The spectral energy density, $u(\omega, \mathbf{r})$, at any location in vacuum can be determined by ${ }^{29}$

$$
u(\omega, \mathbf{r})=\left\langle\frac{\varepsilon_{0}}{2}\left|E_{k}(\omega, \mathbf{r})\right|^{2}+\frac{\mu_{0}}{2}\left|H_{k}(\omega, \mathbf{r})\right|^{2}\right\rangle,
$$

where $\varepsilon_{0}$ and $\mu_{0}$ are the permittivity and permeability of free space. Since the temperatures of objects are assumed to be time invariant, Poynting vector and energy density can be determined from their spectral counterparts using the relation $f(\mathbf{r})=\int_{0}^{\infty} f(\omega, \mathbf{r}) d \omega$, where $f(\omega, \mathbf{r})$ is $P_{i}(\omega, \mathbf{r})$ or $u(\omega, \mathbf{r})$. An important distinction between energy transfer and energy density is that energy transfer between two objects is nonzero only if they are at different temperatures, whereas energy density can be nonzero irrespective of the temperatures of the objects.

While the theory of thermodynamics of near-field radiative transfer should be applicable to objects of arbitrary shapes and sizes, we focus on the case of planar multilayered objects as shown in Fig. 1, since the dyadic Green's functions are well known. ${ }^{30,31}$ The two half-spaces in Fig. 1 to the left and right, $L$ and $R$ respectively, are at temperatures $T_{L}$ and $T_{R}$. Each halfspace, composed of a homogeneous material or multiple planar films, is assumed to be isothermal. Multilayered half-spaces do not introduce any conceptual difficulties and are taken into account via generalized Fresnel reflection coefficients, $\tilde{R}_{0 L}$ and $\tilde{R}_{0 R} \cdot{ }^{30,31}$ Because of translational symmetry in the $x-y$ plane, all quantities of our interest only depend on $z$. By using the theory of fluctuational electrodynamics and the dyadic Green's

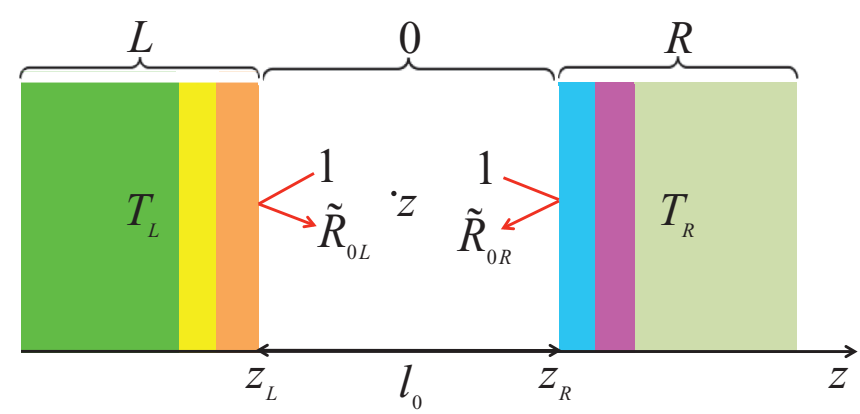

FIG. 1. (Color online) Multilayered half-spaces separated by a vacuum gap of thickness $l_{0}$. Half-spaces can be homogeneous materials or can be composed of planar multilayer stacks. 
function formalism ${ }^{6}$ (Antezza et al. ${ }^{32}$ used the same formalism to calculate the thermal nonequilibrium stress tensor in the vacuum cavity), the integral expressions can be obtained for the energy density due to thermal fluctuations, $u_{h}^{(j)}(z)$, in vacuum due to $j$-polarized electromagnetic waves from half-space $h$ $(h=L, R) . j=s$ stands for transverse electric polarization and $j=p$ stands for transverse magnetic polarization. $u_{L}^{(j)}(z)$ and $u_{R}^{(j)}(z)$ are given by

$$
\begin{aligned}
& u_{L}^{(j)}(z)=\int_{0}^{\infty} \int_{0}^{k_{0}} \int_{0}^{2 \pi} \frac{d k_{0} d k_{\rho} k_{\rho} d \phi}{k_{z 0} / k_{0}} \frac{\Theta\left(\omega, T_{L}\right)}{8 \pi^{3}} \frac{\left(1-\left|\tilde{R}_{0 L}^{(j)}\right|^{2}\right)\left[1+\left|\tilde{R}_{0 R}^{(j)}\right|^{2}+\frac{k_{\rho}^{2}}{k_{0}^{2}} 2 \operatorname{Re}\left(\tilde{R}_{0 R}^{(j)} e^{i 2 k_{z 0}\left(z_{R}-z\right)}\right)\right]}{\left|1-\tilde{R}_{0 L}^{(j)} \tilde{R}_{0 R}^{(j)} e^{i 2 k_{z 0} l_{0}}\right|^{2}} \\
& +\int_{0}^{\infty} \int_{k_{0}}^{\infty} \int_{0}^{2 \pi} \frac{d k_{0} d k_{\rho} k_{\rho} d \phi}{\beta_{z 0} / \sqrt{\beta_{z 0}^{2}+k_{\rho}^{2}}} \frac{k_{0}}{\sqrt{\beta_{z 0}^{2}+k_{\rho}^{2}}} \frac{\Theta\left(\omega, T_{L}\right)}{8 \pi^{3}} \\
& \times \frac{2 \operatorname{Im}\left(\tilde{R}_{0 L}^{(j)}\right) e^{-2 \beta_{z 0} l_{0}}\left[2 \operatorname{Re}\left(\tilde{R}_{0 R}^{(j)}\right)+\frac{k_{\rho}^{2}}{k_{0}^{2}} e^{2 \beta_{z 0}\left(z_{R}-z\right)} \frac{k_{\rho}^{2}}{k_{0}^{2}} e^{-2 \beta_{z 0}\left(z_{R}-z\right)}\left|\tilde{R}_{0 R}^{(j)}\right|^{2}\right]}{\left|1-\tilde{R}_{0 L}^{(j)} \tilde{R}_{0 R}^{(j)} e^{-2 \beta_{z 0} l_{0}}\right|^{2}}, \\
& u_{R}^{(j)}(z)=\int_{0}^{\infty} \int_{0}^{k_{0}} \int_{0}^{2 \pi} \frac{d k_{0} d k_{\rho} k_{\rho} d \phi}{k_{z 0} / k_{0}} \frac{\Theta\left(\omega, T_{R}\right)}{8 \pi^{3}} \frac{\left(1-\left|\tilde{R}_{0 R}^{(j)}\right|^{2}\right)\left[1+\left|\tilde{R}_{0 L}^{(j)}\right|^{2}+\frac{k_{\rho}^{2}}{k_{0}^{2}} 2 \operatorname{Re}\left(\tilde{R}_{0 L}^{(j)} e^{i 2 k_{z 0}\left(z-z_{L}\right)}\right)\right]}{\left|1-\tilde{R}_{0 L}^{(j)} \tilde{R}_{0 R}^{(j)} e^{i 2 k_{z 0} l_{0}}\right|^{2}} \\
& +\int_{0}^{\infty} \int_{k_{0}}^{\infty} \int_{0}^{2 \pi} \frac{d k_{0} d k_{\rho} k_{\rho} d \phi}{\beta_{z 0} / \sqrt{\beta_{z 0}^{2}+k_{\rho}^{2}}} \frac{k_{0}}{\sqrt{\beta_{z 0}^{2}+k_{\rho}^{2}}} \frac{\Theta\left(\omega, T_{R}\right)}{8 \pi^{3}} \\
& \times \frac{2 \operatorname{Im}\left(\tilde{R}_{0 R}^{(j)}\right) e^{-2 \beta_{z 0} l_{0}}\left[2 \operatorname{Re}\left(\tilde{R}_{0 L}^{(j)}\right)+\frac{k_{\rho}^{2}}{k_{0}^{2}} e^{2 \beta_{z 0}\left(z-z_{L}\right)}+\frac{k_{\rho}^{2}}{k_{0}^{2}} e^{-2 \beta_{z 0}\left(z-z_{L}\right)}\left|\tilde{R}_{0 L}^{(j)}\right|^{2}\right]}{\left|1-\tilde{R}_{0 L}^{(j)} \tilde{R}_{0 R}^{(j)} e^{-2 \beta_{z 0} l_{0}}\right|^{2}},
\end{aligned}
$$

where $\mathbf{k}_{\rho}=k_{\rho} \hat{\mathbf{k}}_{\rho}=k_{x} \hat{x}+k_{y} \hat{y}$ is the in-plane wave vector, $k_{0}=\omega / c, k_{\rho}^{2}+k_{z 0}^{2}=k_{0}^{2}$ for propagating waves (PW) ( $\left.0 \leqslant k_{\rho}<k_{0}\right)$, and $k_{\rho}^{2}-\beta_{z 0}^{2}=k_{0}^{2}$ for evanescent waves (EW) $\left(k_{0} \leqslant k_{\rho}<\infty\right) ; l_{0}=\left|z_{R}-z_{L}\right|$ and $\Theta(\omega, T)=\hbar \omega /\left(\exp \left(\hbar \omega / k_{B} T\right)-1\right)$. The square roots of $k_{z 0}$ and $\beta_{z 0}$ are defined so that $k_{z 0} \geqslant 0$ and $\beta_{z 0} \geqslant 0$. The triple integrals are performed over all frequencies or $k_{0}$ and in-plane wave vector space $\left(k_{\rho} \cos \phi, k_{\rho} \sin \phi\right)$ where $0 \leqslant \phi<2 \pi, 0 \leqslant k_{\rho}<k_{0}$ for propagating waves, and $k_{0} \leqslant k_{\rho}<\infty$ for evanescent waves. We use $k_{0}$ instead of $\omega$ so that it has the same physical dimension as $k_{\rho}$. The reason for the unusual form of infinitesimal volume element of integration will be explained shortly in Sec. III A.

The energy flux, which can be obtained from the $z$ component of the Poynting vector, ${ }^{6,33}$ is given by

$\dot{E}_{L}^{(j)}=\int_{0}^{\infty} d k_{0} \frac{c \Theta\left(\omega, T_{L}\right)}{8 \pi^{3}}\left[\int_{0}^{k_{0}} \int_{0}^{2 \pi} d k_{\rho} k_{\rho} d \phi \frac{\left(1-\left|\tilde{R}_{0 L}^{(j)}\right|^{2}\right)\left(1-\left|\tilde{R}_{0 R}^{(j)}\right|^{2}\right)}{\left|1-\tilde{R}_{0 L}^{(j)} \tilde{R}_{0 R}^{(j)} e^{i 2 k_{z 0} l_{0}}\right|^{2}}+\int_{k_{0}}^{\infty} \int_{0}^{2 \pi} d k_{\rho} k_{\rho} d \phi \frac{4 \operatorname{Im}\left(\tilde{R}_{0 L}^{(j)}\right) \operatorname{Im}\left(\tilde{R}_{0 R}^{(j)}\right) e^{-2 \beta_{z 0} l_{0}}}{\left|1-\tilde{R}_{0 L}^{(j)} \tilde{R}_{0 R}^{(j)} e^{-2 \beta_{z 0} l_{0}}\right|^{2}}\right]$,

$\dot{E}_{R}^{(j)}=-\int_{0}^{\infty} d k_{0} \frac{c \Theta\left(\omega, T_{R}\right)}{8 \pi^{3}}\left[\int_{0}^{k_{0}} \int_{0}^{2 \pi} d k_{\rho} k_{\rho} d \phi \frac{\left(1-\left|\tilde{R}_{0 L}^{(j)}\right|^{2}\right)\left(1-\left|\tilde{R}_{0 R}^{(j)}\right|^{2}\right)}{\mid 1-\tilde{R}_{0 L}^{(j)} \tilde{R}_{0 R}^{(j)} e^{\left.i 2 k_{z 0} l_{0}\right|^{2}}}+\int_{k_{0}}^{\infty} \int_{0}^{2 \pi} d k_{\rho} k_{\rho} d \phi \frac{4 \operatorname{Im}\left(\tilde{R}_{0 L}^{(j)}\right) \operatorname{Im}\left(\tilde{R}_{0 R}^{(j)}\right) e^{-2 \beta_{z 0} l_{0}}}{\left|1-\tilde{R}_{0 L}^{(j)} \tilde{R}_{0 R}^{(j)} e^{-2 \beta_{z 0} l_{0}}\right|^{2}}\right]$

The energy flux from sources in $L, \dot{E}_{L}^{(j)}$, is in the positive $z$ direction and that from sources in $R, \dot{E}_{R}^{(j)}$, is in along the negative $z$ direction [hence the negative sign in Eq. (8b)].

The spectral energy density due to thermal fluctuations at any location in thermal equilibrium at temperature $T$ can be written as $u(\omega, \mathbf{r})=\rho_{\omega}(\omega, \mathbf{r}) \Theta(\omega, T) .{ }^{28,34}$ In Planck's work, ${ }^{1}$ the function $\rho_{\omega}(\omega, \mathbf{r})=\omega^{2} / \pi^{2} c^{3}$, independent of $\mathbf{r}$, is the electromagnetic density of states in free space. When near-field effects are important, $\rho_{\omega}(\omega, \mathbf{r})$ becomes position dependent and is known as the (electromagnetic) local density of states. ${ }^{28}$ In vacuum, the local density of states is given by ${ }^{28,34}$

$$
\rho_{\omega}(\omega, \mathbf{r})=\frac{\omega}{\pi c^{2}} \operatorname{Im} \operatorname{Tr}\left[\overline{\overline{\mathbf{G}}}_{e}(\omega ; \mathbf{r}, \mathbf{r})+\overline{\overline{\mathbf{G}}}_{m}(\omega ; \mathbf{r}, \mathbf{r})\right]
$$

where $\operatorname{Tr}(\overline{\overline{\mathbf{G}}})=G_{11}+G_{22}+G_{33}$. Unlike energy density due to thermal fluctuations and energy flux, it does not make sense to integrate $\rho_{\omega}(\omega, \mathbf{r})$ over all frequencies since the integrand increases as $\omega^{2}$ as $\omega \rightarrow \infty$. Since we have chosen the spectral variable to be $k_{0}=\omega / c$ instead of $\omega$, we define a new local density of states $\rho_{k_{0}}\left(k_{0}, \mathbf{r}\right)$ such that $\rho_{k_{0}}\left(k_{0}, \mathbf{r}\right) d k_{0}=\rho_{\omega}(\omega, \mathbf{r}) d \omega$. It can be seen 
that $\rho_{k_{0}}\left(k_{0}, \mathbf{r}\right)=c \rho_{\omega}(\omega, \mathbf{r})$. The polarized $\rho_{k_{0}}^{(j)}\left(k_{0}, z\right)$ is given by

$$
\begin{aligned}
\rho_{k_{0}}^{(j)}\left(k_{0}, z\right)= & \int_{0}^{k_{0}} \int_{0}^{2 \pi} \frac{d k_{\rho} k_{\rho} d \phi}{k_{z 0} / k_{0}} \frac{1}{4 \pi^{3}} \operatorname{Re}\left[\frac{1+\tilde{R}_{0 L}^{(j)} \tilde{R}_{0 R}^{(j)} e^{i 2 k_{z 0} l_{0}}+\frac{k_{\rho}^{2}}{k_{0}^{2}} \tilde{R}_{0 R}^{(j)} e^{i 2 k_{z 0}\left(z_{R}-z\right)}+\frac{k_{\rho}^{2}}{k_{0}^{2}} \tilde{R}_{0 L}^{(j)} e^{i 2 k_{z 0}\left(z-z_{L}\right)}}{1-\tilde{R}_{0 L}^{(j)} \tilde{R}_{0 R}^{(j)} e^{i 2 k_{z 0} l_{0}}}\right] \\
& +\int_{k_{0}}^{\infty} \int_{0}^{2 \pi} \frac{d k_{\rho} k_{\rho} d \phi}{\beta_{z 0} / \sqrt{\beta_{z 0}^{2}+k_{\rho}^{2}}} \frac{k_{0}}{\sqrt{\beta_{z 0}^{2}+k_{\rho}^{2}}} \frac{1}{4 \pi^{3}} \operatorname{Im}\left[\frac{2 \tilde{R}_{0 L}^{(j)} \tilde{R}_{0 R}^{(j)} e^{-2 \beta_{z 0} l_{0}}+\frac{k_{\rho}^{2}}{k_{0}^{2}}\left[\tilde{R}_{0 R}^{(j)} e^{-2 \beta_{z 0}\left(z_{R}-z\right)}+\tilde{R}_{0 L}^{(j)} e^{-2 \beta_{z 0}\left(z-z_{L}\right)}\right]}{1-\tilde{R}_{0 L}^{(j)} \tilde{R}_{0 R}^{(j)} e^{-2 \beta_{z 0} l_{0}}}\right]
\end{aligned}
$$

\section{DETERMINING ENTROPY DENSITY}

Entropy can be described by determining the number of accessible microscopic states into which a given number of photons are distributed because of the presence of near-field effects. Let the space containing the microscopic states into which the electromagnetic waves or photons are distributed be represented by $\mu$. The space $\mu$ that contains the microscopic states is as yet undefined. We will use Eq. (7) to determine $\mu$. Consider a volume within the vacuum cavity of lateral area $A$ (in the $x-y$ plane) lying between coordinates $z$ and $z+d z$. The total number of accessible microscopic states in an infinitesimal element $d \mu$ within the volume $A d z$ of real space is $\rho_{a}(\mu, z) d \mu A d z$. We will refer to $\rho_{a}(\mu, z)$ from now on as "local density of accessible microscopic states." We will show that $\rho_{a}(\mu, z)$ is independent of the half-space under consideration. $\rho_{a}(\mu, z)$ is related to $\rho_{k_{0}}\left(k_{0}, z\right)$ but need not be equal to it. The number of photons from half-space $h(h=L$ or $R$ ) within the states $d \mu$ in the volume $A d z$ is $n_{h}(\mu, z) d \mu A d z$. The entropy associated with the $n_{h}(\mu, z) d \mu A d z$ photons is given by

$$
s_{h}(\mu, z) d \mu A d z=k_{B} \ln \delta W_{h},
$$

where $\delta W_{h}$ is the number of ways in which $n_{h}(\mu, z) d \mu A d z$ photons can be distributed in $\rho_{a}(\mu, z) d \mu A d z$ states. $\delta W_{h}$ is given by ${ }^{35,36}$

$$
\delta W_{h}=\frac{\left(n_{h}(\mu, z) d \mu A d z+\rho_{a}(\mu, z) d \mu A d z-1\right) !}{\left(n_{h}(\mu, z) d \mu A d z\right) !\left(\rho_{a}(\mu, z) d \mu A d z-1\right) !} .
$$

Substituting Eq. (12) into Eq. (11), the polarization dependent entropy density due to temperature $T_{h}$ is

$$
\begin{aligned}
s_{h}^{(j)}(\mu, z)= & k_{B} \rho_{a}^{(j)}(\mu, z)\left[\left(1+\frac{n_{h}^{(j)}(\mu, z)}{\rho_{a}^{(j)}(\mu, z)}\right) \ln \left(1+\frac{n_{h}^{(j)}(\mu, z)}{\rho_{a}^{(j)}(\mu, z)}\right)\right. \\
& \left.-\left(\frac{n_{h}^{(j)}(\mu, z)}{\rho_{a}^{(j)}(\mu, z)}\right) \ln \left(\frac{n_{h}^{(j)}(\mu, z)}{\rho_{a}^{(j)}(\mu, z)}\right)\right] .
\end{aligned}
$$

But for the position-dependent $n_{h}^{(j)}(\mu, z)$ and, as yet undefined, $\rho_{a}^{(j)}(\mu, z)$, Eqs. (13) and (1) are remarkably similar. Since plane waves with different $\mu$ are incoherent, the entropy density due to them must be additive. ${ }^{12,14,15}$ The same can be said of waves generated from the two half-spaces. Hence the total entropy density at any location $z$ is given by

$$
s(z)=\int d \mu \sum_{j=s, p}\left(s_{L}^{(j)}(\mu, z)+s_{R}^{(j)}(\mu, z)\right),
$$

where $s_{L}^{(j)}$ and $s_{R}^{(j)}$ are the entropy contribution due to thermal fluctuations in the left and right half-spaces, respectively. Thermal nonequilibrium entropy density can be evaluated only if we can determine $\mu, n_{h}^{(j)}(\mu, z)$ and $\rho_{a}^{(j)}(\mu, z)$.

\section{A. Determining $\mu, n_{h}^{(j)}(\mu, z)$ and $\rho_{a}^{(j)}(\mu, z)$}

The $\left\{\mathbf{k}_{\rho}, k_{z}\right\}$ or $\left\{\mathbf{k}_{\rho}, \beta_{z}\right\}$ space can be filled by constant $k_{0}$ or constant energy surfaces. Constant $k_{0}$ surfaces, which are spheres for $\mathrm{PW}\left(k_{\rho}^{2}+k_{z}^{2}=k_{0}^{2}\right)$ and hyperboloids for $\mathrm{EW}\left(k_{\rho}^{2}-\right.$ $\beta_{z}^{2}=k_{0}^{2}$ ), are shown in Figs. 2(a) and 2(b), respectively (only $k_{z}, \beta_{z}>0$ portion is shown). The $k_{z}$ and $\beta_{z}$ axes are parallel to the positive $z$ axis. Because of the dispersion relations for plane waves, the constant $k_{0}$ surfaces are double valued. For each value of $\mathbf{k}_{\rho}$, there exist two values of $k_{z}$ or $\beta_{z}$, given by $k_{z 0}= \pm \sqrt{k_{0}^{2}-k_{\rho}^{2}}$ for $k_{\rho} \leqslant k_{0}$ and $\beta_{z 0}= \pm \sqrt{k_{\rho}^{2}-k_{0}^{2}}$ for $k_{\rho}>$ $k_{0}$. Since the expression for energy density (or stress tensor) is an integral over the in-plane wave vectors and frequency, we can identify $\mu$ as the space spanned by $\left\{k_{\rho} \in(0, \infty), \phi \in\right.$ $(0,2 \pi), k_{z}$ or $\left.\beta_{z} \in(-\infty, \infty)\right\}$.

Consider a differential patch on a constant energy surface [blue patches in Figs. 2(a) and 2(b)] whose projected area in the $k_{x}-k_{y}$ plane is $d k_{x} d k_{y}$ or $k_{\rho} d k_{\rho} d \phi$. The area of this blue patch is given by $d S=\frac{k_{\rho} d k_{\rho} d \phi}{\left|\cos \theta_{z}(\mu)\right|}$, where $\theta_{z}(\mu)$ is the angle between the surface normal to the constant energy surface and the $k_{z}$ or $\beta_{z}$ axis. $\cos \theta_{z}(\mu)=k_{z 0} / k_{0}$ for propagating waves and $\cos \theta_{z}(\mu)=\beta_{z 0} / \sqrt{\beta_{z 0}^{2}+k_{\rho}^{2}}$ for evanescent waves. $d \mu$ is an infinitesimal volume element of area $d S$ on a constant $k_{0}$ surface and thickness $d k_{0}$ perpendicular to it. The magnitude (a)

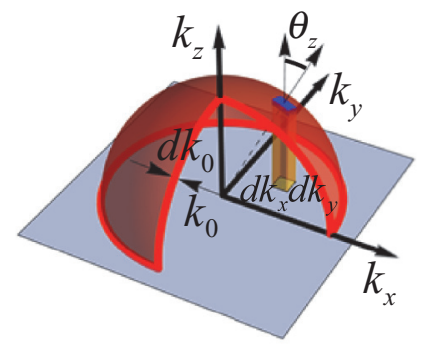

(b)

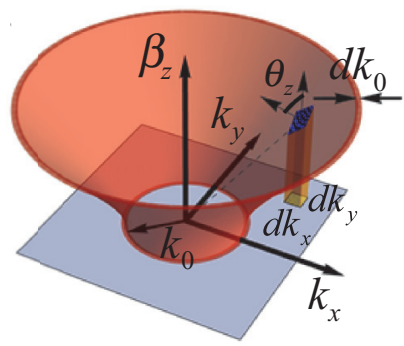

FIG. 2. (Color online) Constant frequency surfaces in upper halfspace for (a) propagating waves $\left(0 \leqslant k_{\rho}<k_{0}\right)$ and (b) evanescent waves $\left(k_{\rho} \geqslant k_{0}\right)$. 
of $d \mu$ is $d k_{0} d S$, given by

$$
d \mu=\frac{d k_{0} d k_{\rho} k_{\rho} d \phi}{\left|\cos \theta_{z}(\mu)\right|}= \begin{cases}\frac{d k_{0} d k_{\rho} k_{\rho} d \phi}{\left|k_{z 0}\right| / k_{0}} & \text { for } \mathrm{PW}, \\ \frac{d k_{0} d k_{\rho} k_{\rho} d \phi}{\left|\beta_{z 0}\right| / \sqrt{\beta_{z 0}^{2}+k_{\rho}^{2}}} & \text { for } \mathrm{EW} .\end{cases}
$$

At any location $z, n_{h}^{(j)}(\mu, z)$ can be related to $u_{h}^{(j)}(z)$ [defined already in Eq. (7)] as follows:

$$
u_{h}^{(j)}(z)=\int_{\mathrm{PW}} d \mu \hbar \omega n_{h}^{(j)}(\mu, z)+\int_{\mathrm{EW}} d \mu \hbar \omega n_{h}^{(j)}(\mu, z),
$$

where PW and EW stand for "propagating wave" and "evanescent wave," respectively, $\hbar \omega$ is the energy of a photon at frequency $\omega$, and $\int_{\mathrm{PW}} d \mu$ and $\int_{\mathrm{EW}} d \mu$ are defined as follows:

$$
\begin{array}{r}
\int_{\mathrm{PW}} d \mu f(\mu)=\int_{0}^{\infty} \int_{0}^{k_{0}} \int_{0}^{2 \pi} \frac{d k_{0} d k_{\rho} k_{\rho} d \phi}{\left|k_{z 0}\right| / k_{0}} f(\mu), \\
\int_{\mathrm{EW}} d \mu f(\mu)=\int_{0}^{\infty} \int_{k_{0}}^{\infty} \int_{0}^{2 \pi} \frac{d k_{0} d k_{\rho} k_{\rho} d \phi}{\left|\beta_{z 0}\right| / \sqrt{\beta_{z 0}^{2}+k_{\rho}^{2}}} f(\mu),
\end{array}
$$

for any function $f(\mu)$. Since integrals of the form $\int_{\mathrm{PW}} d \mu f(\mu)+\int_{\mathrm{EW}} d \mu f(\mu)$ are necessary to determine energy and entropy density, we will define $\int d \mu f(\mu)$ as

$$
\int d \mu f(\mu)=\int_{\mathrm{PW}} d \mu f(\mu)+\int_{\mathrm{EW}} d \mu f(\mu) .
$$

By comparison with Eq. (7), $n_{L}^{(j)}(\mu, z)$ and $n_{R}^{(j)}(\mu, z)$ are given by

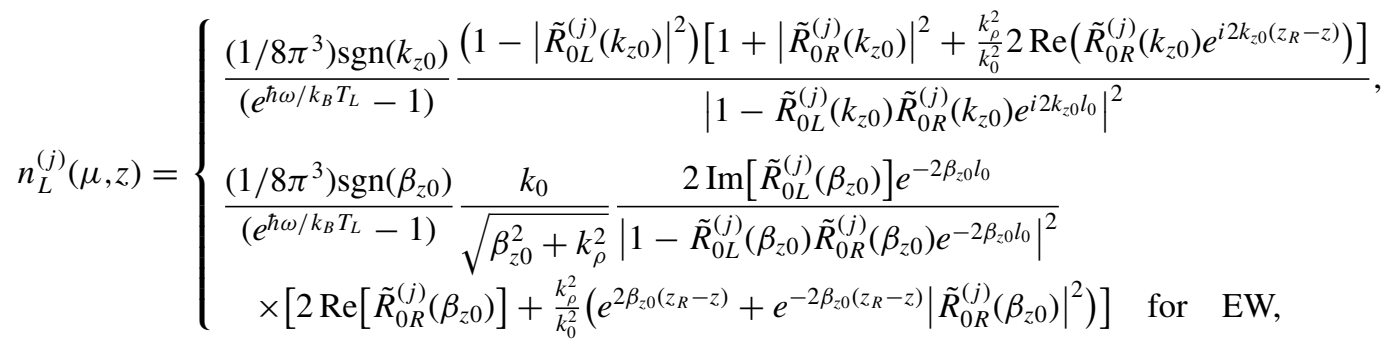

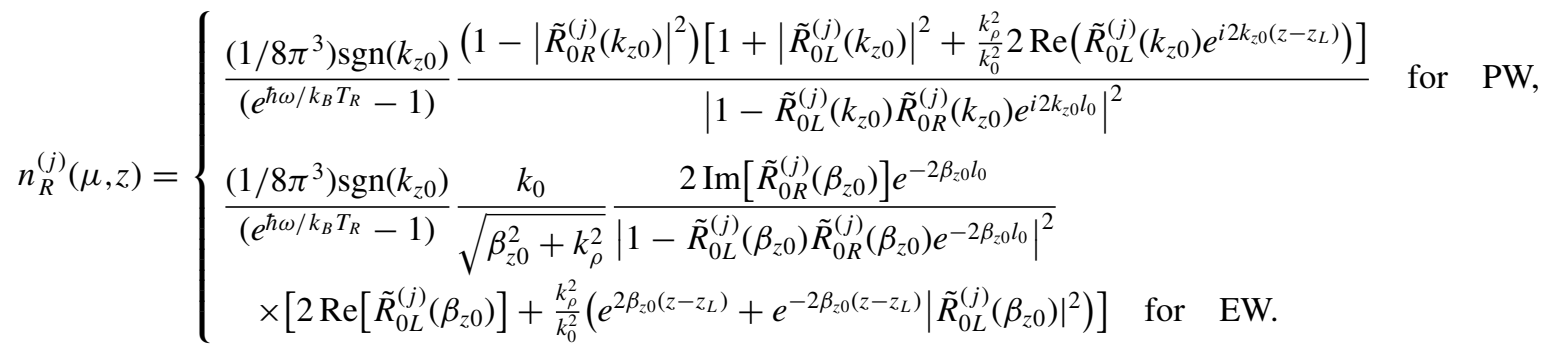

That $n_{L}^{(j)}(\mu, z)$ and $n_{R}^{(j)}(\mu, z)$ are given by Eqs. (20a) and (20b) for $k_{z 0}<0$ or $\beta_{z 0}<0$ can be seen from the following identities: ${ }^{30,37}$

$$
\begin{array}{lll}
\tilde{R}_{0 h}^{(j)}\left(k_{z 0}\right)=\tilde{R}_{0 h}^{(j)-1}\left(\left|k_{z 0}\right|\right) & \text { for } & \mathrm{PW} \\
\tilde{R}_{0 h}^{(j)}\left(\beta_{z 0}\right)=\tilde{R}_{0 h}^{(j)-1}\left(\left|\beta_{z 0}\right|\right) & \text { for } & \mathrm{EW} .
\end{array}
$$

In Eq. (21), all wave vectors other than $k_{z 0}, \beta_{z 0}$ are left unchanged. Since Eqs. (20a) and (20b) are valid for both $k_{z 0}, \beta_{z 0}>0$ as well as $k_{z 0}, \beta_{z 0}<0, n_{L}^{(j)}(\mu, z)$ and $n_{R}^{(j)}(\mu, z)$ can be associated with either surface. Since the Poynting vector due to thermal sources in $L$ and $R$ points in the positive and negative $z$ directions respectively, at any location in the vacuum gap, we will associate $n_{L}^{(j)}(\mu, z)$ and $n_{R}^{(j)}(\mu, z)$ with the $k_{z 0}, \beta_{z 0}>0$ and $k_{z 0}, \beta_{z 0}<0$ portions of the $\mu$ space, respectively.

The local density of states, $\rho_{k_{0}}^{(j)}\left(k_{0}, z\right)$, can also be split into a local density of microscopic states, $\rho_{\mu}^{(j)}(\mu, z)$, that is related to $\rho_{k_{0}}^{(j)}\left(k_{0}, z\right)$ as

$$
\rho_{k_{0}}^{(j)}\left(k_{0}, z\right)=\int_{\mathrm{PW}} d S \rho_{\mu}^{(j)}(\mu, z)+\int_{\mathrm{EW}} d S \rho_{\mu}^{(j)}(\mu, z),
$$

where $\int_{\mathrm{PW}} d S$ and $\int_{\mathrm{EW}} d S$ are defined as follows:

$$
\begin{gathered}
\int_{\mathrm{PW}} d S f(\mu)=\int_{0}^{k_{0}} \int_{0}^{2 \pi} \frac{d k_{\rho} k_{\rho} d \phi}{\left|k_{z 0}\right| / k_{0}} f(\mu), \\
\int_{\mathrm{EW}} d S f(\mu)=\int_{k_{0}}^{\infty} \int_{0}^{2 \pi} \frac{d k_{\rho} k_{\rho} d \phi}{\left|\beta_{z 0}\right| / \sqrt{\beta_{z 0}^{2}+k_{\rho}^{2}}} f(\mu)
\end{gathered}
$$


for any function $f(\mu)$. In parallel with $\int d \mu f(\mu)$, we will define $\int d S f(\mu)$ as

$$
\int d S f(\mu)=\int_{\mathrm{PW}} d S f(\mu)+\int_{\mathrm{EW}} d S f(\mu) .
$$

From Eq. (10), $\rho_{\mu}^{(j)}(\mu, z)$ can be identified as

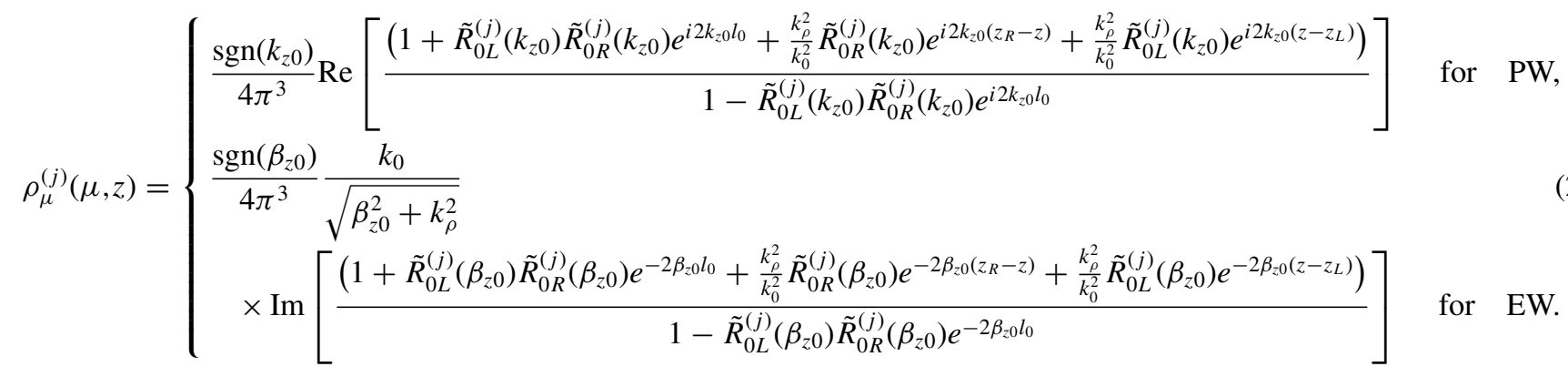

It can be seen from Eq. (26) that, as with $n_{L}^{(j)}(\mu, z)$ and $n_{R}^{(j)}(\mu, z)$, the expression for $\rho_{\mu}^{(j)}(\mu, z)$ is valid irrespective of the sign of $k_{z 0}$ and $\beta_{z 0}$.

$n_{L}^{(j)}(\mu, z)$ and $n_{R}^{(j)}(\mu, z)$ are dependent on temperatures $T_{L}$ and $T_{R}$, respectively, while $\rho_{\mu}^{(j)}(\mu, z)$ is temperature independent. This is because $n_{h}^{(j)}(\mu, z)$ is related to the energy density at $z$ due to thermally fluctuating sources in half-space $h$ at temperature $T_{h} . \rho_{\mu}^{(j)}(\mu, z)$, on the other hand, is a property of electromagnetic waves for the given configuration of objects and is not dependent on the temperature distribution anywhere $^{28,34}$ (all material properties are assumed to be temperature independent).

Two questions still need to be answered: (1) is $\rho_{\mu}^{(j)}(\mu, z)=$ $\rho_{a}^{(j)}(\mu, z)$; ; (2) why are $n_{L}^{(j)}(\mu, z)$ and $n_{R}^{(j)}(\mu, z)$ used separately to determine $s_{L}^{(j)}(\mu, z)$ and $s_{R}^{(j)}(\mu, z)$, respectively? Why is the entropy density not simply determined by taking $n_{L}^{(j)}(\mu, z)+$ $n_{R}^{(j)}(\mu, z)$ into consideration?

We now show that $\rho_{a}^{(j)}(\mu, z)=\rho_{\mu}^{(j)}(\mu, z) / 2$ in the case of thermal nonequilibrium. $\rho_{\mu}^{(j)}(\mu, z)$ is defined, according to Eqs. (22) and (26), as an integrand over the projected area of the constant $k_{0}$ surface on the $\mathbf{k}_{\rho}$ plane. Since both halves of the constant $k_{0}$ surface correspond to the same projected area on the $\mathbf{k}_{\rho}$ plane, $\rho_{\mu}^{(j)}(\mu, z)$ should be divided equally between them. Hence $\rho_{a}^{(j)}(\mu, z)=\rho_{\mu}^{(j)}(\mu, z) / 2$. The case of thermal equilibrium should be treated differently and is discussed in Sec. V.

To justify that entropy density $s_{h}^{(j)}(\mu, z)$ in thermal nonequilibrium $\left(T_{L} \neq T_{R}\right)$ can then be determined by substituting $n_{h}^{(j)}(\mu, z)$ and $\rho_{a}^{(j)}(\mu, z)$ into Eq. (13), we draw on the works of von Laue ${ }^{12}$ and Barakat ${ }^{14,15}$ (von Laue's paper, which is in German, should be translated to English so that much-deserved credit can be attributed to his work). Barakat showed that the entropy of two incoherent beams should be additive. Let us take into consideration the two plane waves with the same $\mathbf{k}_{\rho}$ and $k_{0}$ but originating from $L$ and $R$. The plane wave generated from half-space $h(h=L, R)$ is the result of all thermal sources within half-space $h$ alone. These two plane waves, despite the same value of $\mathbf{k}_{\rho}$ and $k_{0}$ are incoherent because they are generated by thermal fluctuations in different objects. Hence the entropy of the two plane waves should be the sum of the entropy of each of the plane waves. This property of entropy density can be satisfied only if $n_{L}^{(j)}(\mu, z)$ and $n_{R}^{(j)}(\mu, z)$ are taken separately to evaluate $s_{L}^{(j)}(\mu, z)$ and $s_{R}^{(j)}(\mu, z)$, respectively.

Zhang and $\mathrm{Basu}^{7}$ have also considered the problem of entropy transfer by thermal radiation between two diffuse and gray ${ }^{8,9}$ (emissivity less than 1) half-spaces at different temperatures in the far-field limit. The reader will realize that the prediction of our theory, in the far-field limit, for entropy transfer is in contradiction with that of Zhang and Basu. ${ }^{7}$ The difference in results is because of the differences in assumptions between our work and that of Zhang and Basu. ${ }^{7}$ Our assumption is that the interfaces are perfectly smooth and planar surfaces at which waves undergo coherent scattering. Zhang and $\mathrm{Basu}^{7}$ assume that photons undergo diffuse and incoherent scattering at the interface.

\section{DETERMINING ENTROPY FLUX}

\section{A. Generalized spectral specific intensity}

We know from the theory of blackbody radiation that the concept of spectral specific intensity of thermal radiation is used extensively to compute energy and entropy fluxes [see Eqs. (1) and (2)]. In trying to extend the calculation to include near-field effects, we partially resolve the following vexing question in the theory of radiative transfer: what is the equivalent of radiation intensity in a field theory, such as the theory of electromagnetic fields governed by Maxwell's equation? In Maxwell's equation, the flux at any point is governed by the Poynting vector and there is no necessity of an intensitylike term. ${ }^{38}$ Though some progress has been made in reconciling radiative transfer and Maxwell's equations by using the concept of energy streamlines, ${ }^{39,40}$ a concrete definition of an intensitylike quantity is still unavailable.

Just as entropy density is obtained from energy density in Sec. III, determining entropy flux requires knowledge of energy flux, which is obtained from the Poynting vector. The polarized energy flux from half-space $h$ can be rewritten [from Eq. (8)] in $\mu$ space for the purpose of introducing generalized 
intensity as ${ }^{6}$

$$
\dot{E}_{h}^{(j)}=\int d \mu \frac{c}{8 \pi^{3}} \cos \theta_{z}(\mu) \Theta\left(\omega, T_{h}\right) \mathcal{T}^{(j)}(\mu),
$$

where $\mathcal{T}^{(j)}(\mu)$ is given by

$$
\mathcal{T}^{(j)}(\mu)=\left\{\begin{array}{l}
\frac{\left(1-\left|\tilde{R}_{0 L}^{(j)}\right|^{2}\right)\left(1-\left|\tilde{R}_{0 R}^{(j)}\right|^{2}\right)}{\left|1-\tilde{R}_{0 L}^{(j)} \tilde{R}_{0 R}^{(j)} e^{i 2 k_{z 0} l_{0}}\right|^{2}} \text { for } \mathrm{PW}, \\
\frac{4 \operatorname{Im}\left(\tilde{R}_{0 L}^{(j)}\right) \operatorname{Im}\left(\tilde{R}_{0 R}^{(j)}\right) e^{-2 \beta_{z 0} l_{0}}}{\mid 1-\tilde{R}_{0 L}^{(j)} \tilde{R}_{0 R}^{(j)} e^{-\left.2 \beta_{z 0} l_{0}\right|^{2}}} \text { for EW. }
\end{array}\right.
$$

Because $\cos \theta_{z}(\mu)>0$ when $k_{z 0}, \beta_{z 0}>0$ and $\cos \theta_{z}(\mu)<0$ when $k_{z 0}, \beta_{z 0}<0, \dot{E}_{L}^{(j)}>0$ and $\dot{E}_{R}^{(j)}<0$, in agreement with Eqs. (8a) and (8b). $\mathcal{T}^{(j)}(\mu)$ in Eq. (28) can be interpreted as a generalized transmissivity $6,33,41$ in vacuum between two multilayered half-spaces $L$ and $R$. Here, we can define a generalized spectral specific intensity of thermal radiation, valid for propagating waves as well as evanescent waves, as

$$
I^{(j)}\left(\mu, T_{h}\right)=\frac{k_{0}^{2}}{8 \pi^{3}} \Theta\left(\omega, T_{h}\right) \mathcal{T}^{(j)}(\mu) .
$$

The concept of specific intensity in classical theory of thermal radiation is associated with the power contained in a cone of $d \Omega$. For propagating waves, $d S / k_{0}^{2}$ can be associated with the solid angle in the direction of propagation because the constant $k_{0}$ surface happens to be spherical. The solid angle interpretation of intensity is invalid for evanescent waves.

$I^{(j)}(\mu)$ on the positive half of a constant $k_{0}$ surface for propagating waves and evanescent waves (the entire surface is not shown) is shown in Figs. 3(a) and 3(b), respectively (simulation performed for $s$ polarization, $l_{0}=5 \mu \mathrm{m}, 2 \pi / k_{0}=$ $10 \mu \mathrm{m}$, and $\varepsilon_{L}=\varepsilon_{R}=2.2+0.01 i$ ). In each of the two figures, the length of the arrows are proportional to the magnitude of $I^{(j)}(\mu)$. The arrows are perpendicular to the constant $k_{0}$ surface. As $k_{\rho} \rightarrow \infty$, the length of the arrows decreases exponentially, in accordance with Eq. (28).

\section{B. Entropy flux}

Energy flux $\dot{E}_{h}^{(j)}$ can be also expressed as the product of energy density and energy transmission velocity as

$$
\dot{E}_{h}^{(j)}=\int d \mu \hbar \omega n_{h}^{(j)}(\mu, z) v_{e, h}^{(j)}(\mu, z),
$$
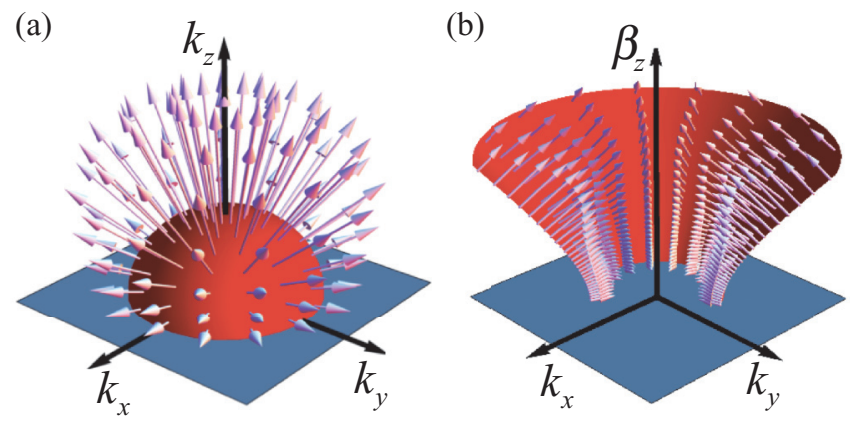

FIG. 3. (Color online) Generalized polarized radiation intensity on constant frequency surfaces for (a) propagating waves and (b) evanescent waves. where $v_{e, h}^{(j)}(\mu, z)$ is the $z$ component of the polarization dependent local velocity of energy transmission associated with photons from half-space $h$. By using Eqs. (20), (27), and (28), we can show that $v_{e, h}^{(j)}(\mu, z)$ is given by

$$
v_{e, h}^{(j)}(\mu, z)=\frac{c k_{z 0}}{k_{0}} \frac{\frac{1-\left|\tilde{R}_{0 \bar{h}}^{(j)}\left(\left|k_{z 0}\right|\right)\right|^{2}}{1+\left|\tilde{R}_{0 \bar{h}}^{(j)}\left(\left|k_{z 0}\right|\right)\right|^{2}}}{1+\frac{k_{\rho}^{2}}{k_{0}^{2}} \frac{2 \operatorname{Re}\left(\tilde{R}_{0 \bar{h}}^{(j)}\left(\left|k_{z 0}\right|\right) e^{i 2\left|k_{z 0}\right| l_{\bar{h}}}\right)}{1+\left|\tilde{R}_{0 \bar{h}}^{(j)}\left(\left|k_{z 0}\right|\right)\right|^{2}}}
$$

for propagating waves, and

$$
v_{e, h}^{(j)}(\mu, z)=\frac{\frac{c \beta_{z 0} k_{0}}{k_{\rho}^{2}} \frac{2 \operatorname{Im}\left[\tilde{R}_{0 \bar{h}}^{(j)}\left(\left|\beta_{z 0}\right|\right)\right] e^{-2\left|\beta_{z 0}\right| l_{\bar{h}}}}{1+\left|\tilde{R}_{0 \bar{h}}^{(j)}\left(\left|\beta_{z 0}\right|\right)\right|^{2} e^{-4\left|\beta_{z 0}\right| l_{\bar{h}}}}}{1+\frac{k_{0}^{2}}{k_{\rho}^{2}} \frac{2 \operatorname{Re}\left[\tilde{R}_{0 \bar{h}}^{(j)}\left(\left|\beta_{z 0}\right|\right)\right] e^{-2\left|\beta_{z 0}\right| l_{\bar{h}}}}{1+\left|\tilde{R}_{0 \bar{h}}^{(j)}\left(\left|\beta_{z 0}\right|\right)\right|^{2} e^{-4\left|\beta_{z 0}\right| l_{\bar{h}}}}}
$$

for evanescent waves. In Eqs. (31) and (32), $\bar{h}=R$ if $h=$ $L$ and $\bar{h}=L$ if $h=R ; l_{L}=z-z_{L}$ and $l_{R}=z_{R}-z$. The $z$ component of the energy transmission velocity $v_{e, h}^{(j)}(\mu, z)$ of electromagnetic waves from half-space $h$ depends only the reflection coefficient $\tilde{R}_{0 \bar{h}}^{(j)}$ at the vacuum $-\bar{h}$ interface, thereby ensuring that $v_{e, h}^{(j)}(\mu, z)$ is given by $c k_{z 0} / k_{0}$ when $R_{0 \bar{h}}=0$. Hence, as expected, the energy transmission velocity of waves emitted from a half-space into vacuum is given by $c$. We have also confirmed numerically that $v_{e, h}^{(j)}(\mu, z) \leqslant c$ for all $\mu$.

Entropy flux associated with this energy flux [Eq. (30)] is given by

$$
\dot{S}_{h}^{(j)}(z)=\int d \mu s_{h}^{(j)}(\mu, z) v_{e, h}^{(j)}(\mu, z) .
$$

Unlike energy flux in the cavity which is $z$ independent, though any two waves with different $\mu$ are incoherent, interference effects due to multiple reflections of the same wave lead to a $z$-dependent entropy flux. ${ }^{14}$

\section{DISCUSSION}

\section{A. Entropy at thermal equilibrium}

At thermal equilibrium, the temperature of the entire system is uniform, namely $T_{L}=T_{R}=T$. The equilibrium case has to be treated differently since emissions from both half-spaces are at the same temperature. When $T_{L}=T_{R}$, electromagnetic waves in the vacuum cavity cannot be distinguished as originating from $L$ or $R$ because their temperatures are equal. Hence it is $n_{\text {tot }}^{(j)}(\mu, z)=n_{L}^{(j)}(\mu, z)+n_{R}^{(j)}(\mu, z)$, rather than $n_{L}^{(j)}(\mu, z)$ or $n_{R}^{(j)}(\mu, z)$ independently, that determines the entropy. Here $\mu=\left\{\mathbf{k}_{\rho},\left|k_{z 0}\right|\right\}$ or $\left\{\mathbf{k}_{\rho},\left|\beta_{z 0}\right|\right\}$. Since we have adopted the convention that $n_{R}^{(j)}(\mu, z)$ is allocated to the $k_{z 0}, \beta_{z 0}<0$ portion of $\mu$ space, $n_{R}$ corresponding to $n_{L}^{(j)}(\mu, z)$ should be evaluated at $\mu_{-}=\left\{\mathbf{k}_{\rho},-\left|k_{z 0}\right|\right\}$ or $\left\{\mathbf{k}_{\rho},-\left|\beta_{z 0}\right|\right\}$. However, we have also shown using Eq. (21) that we obtain the same value for $n_{R}$ irrespective of using $\mu$ or $\mu_{-}$. Hence we have used $\mu$ instead of $\mu_{-}$to evaluate $n_{R}$. Since $k_{z}, \beta_{z} \geqslant 0$ and $k_{z}, \beta_{z} \leqslant 0$ portions of the constant $k_{0}$ surface are accessible now, i.e., the photons at $\mu$ and $\mu_{-}$are considered together, 
$\rho_{a}^{(j)}(\mu, z)=\rho_{\mu}^{(j)}(\mu, z)$. By substituting $n_{\text {tot }}^{(j)}(\mu, z)$ and $\rho_{\mu}^{(j)}(\mu, z)$ for $n_{h}^{(j)}(\mu, z)$ and $\rho_{a}^{(j)}(\mu, z)$, respectively, in Eq. (13), we obtain the following expression for $s^{(j)}(\mu, z)$ in thermal equilibrium:

$$
\begin{aligned}
s_{\text {eq }}^{(j)}(\mu, z)= & k_{B} \rho_{\mu}^{(j)}(\mu, z)\left[-\frac{n_{\text {tot }}^{(j)}(\mu, z)}{\rho_{\mu}^{(j)}(\mu, z)} \ln \left(\frac{n_{\text {tot }}^{(j)}(\mu, z)}{\rho_{\mu}^{(j)}(\mu, z)}\right)\right. \\
& \left.+\left(1+\frac{n_{\text {tot }}^{(j)}(\mu, z)}{\rho_{\mu}^{(j)}(\mu, z)}\right) \ln \left(1+\frac{n_{\text {tot }}^{(j)}(\mu, z)}{\rho_{\mu}^{(j)}(\mu, z)}\right)\right],
\end{aligned}
$$

where the subscript eq denotes equilibrium.

The equilibrium energy density in the vacuum cavity can be obtained from $\hbar \omega n_{\text {tot }}^{(j)}(\mu, z)$. Using Eqs. (20a), (20b), and (26), it can be shown that

$$
\hbar \omega n_{\text {tot }}^{(j)}(\mu, z)=\rho_{\mu}^{(j)}(\mu, z) \Theta(\omega, T) .
$$

Hence Eq. (34) for $s_{\text {eq }}^{(j)}(\mu, z)$ can be simplified as ${ }^{1,20}$

$$
s_{\mathrm{eq}}^{(j)}(\mu, z)=\rho_{\mu}^{(j)}(\mu, z) \Gamma(\omega, T),
$$

where

$$
\begin{aligned}
\Gamma(\omega, T) & =\frac{\hbar \omega / T}{e^{\hbar \omega / k_{B} T}-1}-k_{B} \ln \left(1-e^{-\hbar \omega / k_{B} T}\right) \\
& =\frac{\partial}{\partial T}\left[k_{B} T \ln \left[2 \sinh \left(\frac{\hbar \omega}{2 k_{B} T}\right)\right]\right] .
\end{aligned}
$$

By integrating both sides of Eq. (36) according to Eq. (25), and summing over both polarizations, we obtain the following expression for the spectral local entropy density:

$$
s_{\omega, \text { eq }}(\omega, z)=\rho_{\omega}(\omega, z) \Gamma(\omega, T) .
$$

The expression for equilibrium spectral entropy density in Eq. (38) is in agreement with Dorofeyev's prediction. ${ }^{20}$ Unlike Dorofeyev's method, which is applicable only for thermal equilibrium conditions, our method treats the equilibrium problem as a special case of the nonequilibrium problem.

We can go further than Dorofeyev and enquire what happens to the entropy flux at thermal equilibrium. Of $s_{\mathrm{eq}}^{(j)}(\mu, z)$, a fraction $n_{L}^{(j)}(\mu, z) / n_{\text {tot }}^{(j)}(\mu, z)$ is from half-space $L$ and the remainder from $R$. The equilibrium entropy flux in the cavity due to thermal sources within $L$ and $R$ are given by

$$
\dot{S}_{L}^{\mathrm{eq}}=\int d \mu \sum_{j=s, p} s_{\mathrm{eq}}^{(j)}(\mu, z) \frac{n_{L}^{(j)}(\mu, z)}{n_{\mathrm{tot}}^{(j)}(\mu, z)} v_{e, L}^{(j)}(\mu, z)
$$

and

$$
\dot{S}_{R}^{\mathrm{eq}}=\int d \mu \sum_{j=s, p} s_{\mathrm{eq}}^{(j)}(\mu, z) \frac{n_{R}^{(j)}(\mu, z)}{n_{\mathrm{tot}}^{(j)}(\mu, z)} v_{e, R}^{(j)}(\mu, z) .
$$

Since $n_{R}^{(j)}(\mu, z) v_{e, R}^{(j)}(\mu, z)=-n_{L}^{(j)}(\mu, z) v_{e, L}^{(j)}(\mu, z)$ at thermal equilibrium [as can be seen from Eq. (30)], $\dot{S}_{L}^{\text {eq }}$ and $\dot{S}_{R}^{\text {eq }}$ cancel each other and the net entropy flux is, as required of thermal equilibrium, zero.

\section{B. Agreement with theory of blackbody radiation}

Here, we compare the prediction of our theory with that of the theory of blackbody radiation for entropy flux emitted by a blackbody. To do that, the configuration shown in Fig. 1 needs to be modified appropriately so that we have a blackbody at the desired temperature. This can be achieved by making half-space $L$ a medium with complex refractive index $1+i \delta$ $(\delta \rightarrow 0)$, so that $\tilde{R}_{0 L}=0$. Since no incident radiation on $L$ is reflected $\left(\tilde{R}_{0 L}=0\right)$ and all radiation is absorbed by $L$ (because of infinite depth and finite but small $\delta$ ), $L$ by definition is a blackbody. We also assume that $\tilde{R}_{0 R}=0$ so that we have a blackbody emitting into vacuum. So, $n_{L}^{(j)}(\mu, z)$ is given by

$$
n_{L}^{(j)}(\mu, z)=\left\{\begin{array}{lll}
\frac{1 / 8 \pi^{3}}{e^{\hbar \omega / k_{B} T_{L}}-1} & \text { for } & \mathrm{PW}, \\
0 & \text { for } & \mathrm{EW}
\end{array}\right.
$$

and $\rho_{a}^{(j)}(\mu, z)$ is given by

$$
\rho_{a}^{(j)}(\mu, z)=\left\{\begin{array}{lll}
\frac{1}{8 \pi^{3}} & \text { for } & \mathrm{PW}, \\
0 & \text { for } & \mathrm{EW} .
\end{array}\right.
$$

Calculating themal nonequilibrium entropy density using Eq. (13), $s_{L}^{(j)}(\mu, z)$ is given by

$$
s_{L}^{(j)}(\mu, z)=\left\{\begin{array}{lll}
\frac{1}{8 \pi^{3}} \Gamma\left(\omega, T_{L}\right) & \text { for } & \mathrm{PW}, \\
0 & \text { for } & \mathrm{EW},
\end{array}\right.
$$

where $\Gamma\left(\omega, T_{L}\right)$ is defined in Eq. (37). Comparison of Eqs. (36) and (43) shows that the nonequilibrium entropy density of blackbody has the same form as the equilibrium entropy density.

The velocity of energy propagation is given by

$$
v_{e, L}^{(j)}(\mu, z)=\left\{\begin{array}{lll}
\frac{c k_{z 0}}{k_{0}} & \text { for } & \mathrm{PW}, \\
0 & \text { for } \mathrm{EW} .
\end{array}\right.
$$

Substituting Eqs. (43) and (44) into Eq. (33), we correctly predict the entropy flux of a blackbody emitter ${ }^{1,11}$ as

$$
\dot{S}_{L}^{(j)}=\frac{\pi^{2} k_{B}^{4} T_{L}^{3}}{45 \hbar^{2} c^{2}}=\frac{4 \sigma T_{L}^{3}}{3} .
$$

\section{Maximum thermodynamic efficiency in near-field energy conversion}

Finally, we apply our theory to determine the maximum possible efficiency that could be achieved in conversion of thermal energy to work through near-field thermal radiative transfer. The maximum work that can be extracted from nearfield radiative transfer between the two half-spaces (rejecting radiation at sink temperature $T_{R}$ ) in Fig. 1 is given by ${ }^{11}$

$$
\dot{W}_{\max }=\left(\dot{E}_{L}-T_{R} \dot{S}_{L}\right)-\left(\left|\dot{E}_{R}\right|-T_{R}\left|\dot{S}_{R}\right|\right) \text {. }
$$

In this expression, both $\dot{S}_{L}$ and $\dot{S}_{R}$ are evaluated at $z=$ $z_{R}$. The absolute values are used for $\dot{E}_{R}$ and $\dot{S}_{R}$ because they are negative (i.e., in the negative $z$ direction). The maximum efficiency of work extraction is then given by $\eta_{\max }=\dot{W}_{\max } / \dot{E}_{L}$.

Park et $a l .{ }^{4}$ analyzed the performance of a thermophotovoltaic converter using tungsten (W) emitter at $2000 \mathrm{~K}$ and $\mathrm{In}_{0.18} \mathrm{Ga}_{0.82} \mathrm{Sb}$ photovoltaic cell at $300 \mathrm{~K}$. Using optical data ${ }^{42}$ for $\mathrm{W}$ and $\mathrm{In}_{0: 18} \mathrm{Ga}_{0.82} \mathrm{Sb}$, we compute energy and entropy fluxes $\dot{E}_{L}, \dot{S}_{L}, \dot{E}_{R}$, and $\dot{S}_{R}$, of which $\dot{E}_{L}$ and $T_{R} \dot{S}_{L}$ are shown in Fig. 4, when half-space $L$ is $\mathrm{W}$ at $2000 \mathrm{~K}$ and half-space $R$ is $\mathrm{In}_{0.18} \mathrm{Ga}_{0.82} \mathrm{Sb}$ at $300 \mathrm{~K}$. Both energy and entropy fluxes 


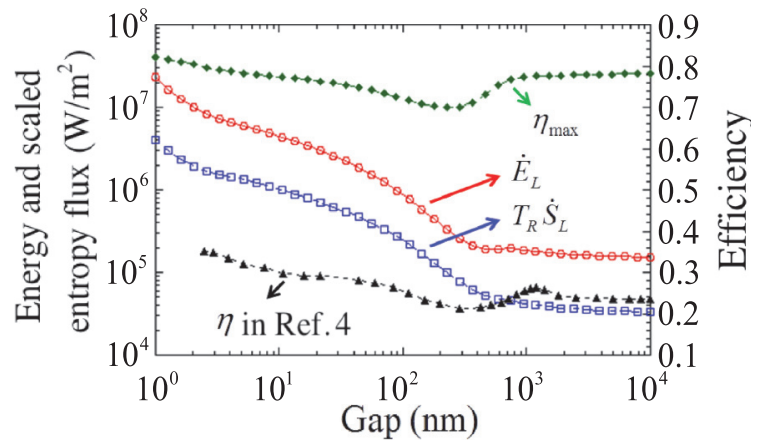

FIG. 4. (Color online) Energy and scaled entropy flux (left $y$ axis, hollow markers) and conversion efficiency (right $y$ axis, solid markers) as a function of vacuum gap for $\mathrm{W}-\mathrm{In}_{0.18} \mathrm{Ga}_{0.82} \mathrm{Sb}$, compared to results from Park et al. ${ }^{4}$

increase as the gap decreases, characteristic of tunneling due to evanescent waves. We also plot efficiency $\eta_{\max }$ as a function of gap and, clearly, the thermodynamic maximum efficiency is much higher than that computed for the model thermophotovoltaic converter. ${ }^{4}$ As observed by Landsberg and Tonge, ${ }^{11}$ though the high values of $\eta_{\max }$ are usually unattainable, the utility of $\eta_{\max }$ is to impose an upper limit on efficiencies of all models for photonic energy conversion, including thermophotovoltaic conversion, involving the same materials, configurations, and temperatures.

\section{CONCLUSION}

Thermodynamic analysis of near-field thermal radiation requires not only the knowledge of energy and momentum, but entropy as well. The knowledge of thermal nonequilibrium entropy transfer between macroscopic objects in near-field radiative transfer enables us to understand such fundamental things as the efficiency of conversion of thermal energy to work via near-field thermal radiative transfer, thermodynamics of surface wave-based laser cooling, ${ }^{43}$ and entropic contributions to nonequilibrium van der Waals/Casimir interactions.

The significance of this work lies in the derivation of a general method to calculate the entropy density and entropy flux in a vacuum cavity between planar multilayered media while taking into account near-field effects. Entropy density and entropy flux are calculated when near-field effects and the condition of thermal nonequilibrium are taken into consideration together. The method is valid for thermal equilbrium condition as well, and agrees with the theory of blackbody radiation in the far-field limit. To compute entropy density and flux, we have defined two new quantities: (1) accessible microscopic states for determining near-field entropy density and (2) generalized spectral intensity of thermal radiation that is valid for both propagating and evanescent waves.

\section{ACKNOWLEDGMENTS}

This work is funded partially by NSF Grant No. CBET0853723 and ONR Grant No. N00014-12-1-0996. *arvind.narayanaswamy@ columbia.edu

${ }^{1}$ M. Planck, The Theory of Heat Radiation (Dover Publications, New York, 2011).

${ }^{2}$ A. Narayanaswamy and G. Chen, Appl. Phys. Lett. 82, 3544 (2003).

${ }^{3}$ M. Laroche, R. Carminati, and J.-J. Greffet, J. Appl. Phys. 100, 063704 (2006).

${ }^{4}$ K. Park, S. Basu, W. P. King, and Z. Zhang, J. Quant. Spectrosc. Radiat. Transfer 109, 305 (2008).

${ }^{5}$ M. Krüger, T. Emig, and M. Kardar, Phys. Rev. Lett. 106, 210404 (2011).

${ }^{6}$ A. Narayanaswamy and Y. Zheng, J. Quant. Spectrosc. Radiat. Transfer (2013), doi:10.1016/j.jqsrt.2013.01.002.

${ }^{7}$ Z. Zhang and S. Basu, Int. J. Heat Mass Transfer 50, 702 (2007).

${ }^{8}$ R. Siegel and J. Howell, Thermal Radiation Heat Transfer (Taylor \& Francis Group, New York, 2002).

${ }^{9}$ M. Modest, Radiative Heat Transfer (Academic Press, New York, 2003).

${ }^{10}$ R. Petela, J. Heat Transfer 86, 187 (1964).

${ }^{11}$ P. Landsberg and G. Tonge, J. Appl. Phys. 51, R1 (1980).

${ }^{12}$ M. Laue, Ann. Phys. (Leipzig) 325, 365 (1906).

${ }^{13}$ A. N. Nigam, Eur. J. Phys. 18, 28 (1999).

${ }^{14}$ R. Barakat, J. Mod. Opt. 30, 1171 (1983).

${ }^{15}$ R. Barakat and C. Brosseau, J. Opt. Soc. Am. A 10, 529 (1993).

${ }^{16}$ A. Rueda, J. Opt. Soc. Am. 63, 573 (1973).

${ }^{17}$ A. J. C. Ladd and W. G. Hoover, Phys. Rev. B 26, 5469 (1982).

${ }^{18}$ S. Kabelac, Proceedings of 5th European Thermal-Sciences Conference, The Netherlands, 2008 (unpublished), pp. 1-12.

${ }^{19}$ S. Kabelac and R. Conrad, Entropy 14, 717 (2012).
${ }^{20}$ I. Dorofeyev, Phys. Scr. 84, 055003 (2011).

${ }^{21}$ A. Pérez-Madrid, L. C. Lapas, and J. M. Rubí, Phys. Rev. Lett. 103, 048301 (2009).

${ }^{22}$ A. Pérez-Madrid, J. M. Rubí, and L. C. Lapas, Phys. Rev. B 77, 155417 (2008)

${ }^{23}$ A. Pérez-Madrid, J. Rubi, and L. Lapas, Mesoscopic NonEquilibrium Thermodynamics: Application to Radiative Heat Exchange in Nanostructures (In-Tech, New York, 2011), Chap. 10, pp. 195-204.

${ }^{24}$ S. Rytov, Tech. Rep., AFCRC-TR-59-162, 1967 (unpublished).

${ }^{25}$ H. Callen and T. Welton, Phys. Rev. 83, 34 (1951).

${ }^{26}$ W. Eckhardt, Opt. Commun. 41, 305 (1982).

${ }^{27}$ Y. Zheng and A. Narayanaswamy, Phys. Rev. A 83, 042504 (2011).

${ }^{28}$ A. Narayanaswamy and G. Chen, J. Quant. Spectrosc. Radiat. Transfer 111, 1877 (2010).

${ }^{29}$ J. A. Stratton, Electromagnetic Theory (Wiley-IEEE Press, New York, 2007), Vol. 33.

${ }^{30}$ W. C. Chew, Waves and Fields in Inhomogenous Media (IEEE Press, New York, 1995).

${ }^{31} \mathrm{~J}$. A. Kong, Electromagnetic Wave Theory (EMW, Cambridge, MA, 2000).

${ }^{32}$ M. Antezza, L. P. Pitaevskii, S. Stringari, and V. B. Svetovoy, Phys. Rev. A 77, 022901 (2008).

${ }^{33}$ S.-A. Biehs, E. Rousseau, and J.-J. Greffet, Phys. Rev. Lett. 105, 234301 (2010).

${ }^{34}$ K. Joulain, R. Carminati, J.-P. Mulet, and J.-J. Greffet, Phys. Rev. B 68, 245405 (2003). 
${ }^{35}$ R. C. Tolman, The Principles of Statistical Mechanics (Dover Publications, New York, 2010).

${ }^{36} \mathrm{Z}$. Zhang, Nano/Microscale Heat Transfer (McGraw-Hill, New York, 2007).

${ }^{37}$ A. Narayanaswamy and Y. Zheng, Phys. Rev. A 88, 012502 (2013).

${ }^{38}$ A. Ishimaru, Wave Propagation and Scattering in Random Media (Wiley-IEEE Press, New York, 1999), Vol. 12.
${ }^{39}$ Z. Zhang and B. Lee, Opt. Express 14, 9963 (2006).

${ }^{40}$ B. Lee, K. Park, and Z. Zhang, Appl. Phys. Lett. 91, 153101 (2007).

${ }^{41}$ P. Ben-Abdallah and K. Joulain, Phys. Rev. B 82, 121419 (2010).

${ }^{42}$ E. D. Palik, Handbook of Optical Constants of Solids (Academic Press, New York, 1998), Vol. 3.

${ }^{43}$ J. B. Khurgin, Phys. Rev. Lett. 98, 177401 (2007). 